\title{
The conceptual cueing database: Rated items for the study of the interaction between language and attention
}

\author{
Stephanie C. Goodhew ${ }^{1,2}$ - Evan Kidd ${ }^{1,2}$ \\ Published online: 11 July 2015 \\ (C) Psychonomic Society, Inc. (outside the USA) 2015
}

\begin{abstract}
Humans appear to rely on spatial mappings to describe and represent concepts. In particular, conceptual cueing refers to the effect whereby after reading or hearing a particular word, the location of observers' visual attention in space can be systematically shifted in a particular direction. For example, words such as "sun" and "happy" orient attention upwards, whereas words such as "basement" and "bitter" orient attention downwards. This area of research has garnered much interest, particularly within the embodied cognition framework, for its potential to enhance our understanding of the interaction between abstract cognitive processes such as language and basic visual processes such as attention and stimulus processing. To date, however, this area has relied on subjective classification criteria to determine whether words ought to be classified as having a meaning that implies "up" or "down." The present study, therefore, provides a set of 498 items that have each been systematically rated by over 90 participants, providing refined, continuous measures of the extent to which people associate given words with particular spatial dimensions. The resulting database provides an objective means to aid item-selection for future research in this area.
\end{abstract}

Keywords Language - Metaphor · Visual attention · Cueing · Conceptual cueing $\cdot$ Conceptual metaphor $\cdot$ Embodied cognition $\cdot$ Embodied semantics

Stephanie C. Goodhew

Stephanie.Goodhew@anu.edu.au

1 Research School of Psychology, The Australian National University, Building 39, Canberra, ACT 0200, Australia

2 Australian Research Council Centre of Excellence for the Dynamics of Language, Canberra, ACT, Australia
There is considerable interaction between abstract cognitive processes on the one hand and one's body and external environment on the other. For example, memory is dramatically enhanced by mentally representing content in familiar spatial locations (Maguire, Valentine, Wilding, \& Kapur, 2003), insight problem solving is facilitated by prescribed physical movements (Thomas \& Lleras, 2007, 2009), and visual awareness of an object is affected by proximity of the observer's hands (Goodhew, Edwards, Ferber, \& Pratt, 2015; Goodhew, Gozli, Ferber, \& Pratt, 2013). Furthermore, humans appear to draw on concrete spatial layouts in order to describe and represent concepts (e.g., Boroditsky, Fuhrman, \& McCormick, 2011). For example, English speakers describe someone who is sad as down, describe improvement as things looking up, and we look forward to the future or back to the past.

A growing body of studies documents the entwined relationship between concepts and space, in particular, how activating word meaning can systematically shift visual attention in space (e.g., Ansorge, Khalid, \& Konig, 2013; Chasteen, Burdzy, \& Pratt, 2010; Dudschig, De la Vega, \& Kaup, 2015; Dudschig, Souman, Lachmair, de la Vega, \& Kaup, 2013; Estes, Verges, \& Barsalou, 2008; Fischer, Castel, Dodd, \& Pratt, 2003; Gozli, Chow, Chasteen, \& Pratt, 2013; Louwerse \& Jeuniaux, 2010; Meier \& Robinson, 2004; Santiago, Lupianez, Perez, \& Funes, 2007; Setic \& Domijan, 2007; Weger \& Pratt, 2008; Zwaan \& Yaxley, 2003). For example, after reading a word associated with $u p$ (such as "sun" or "joy"), participants are faster to respond to subsequent visual targets above the center of the screen and slower to respond to targets below the center, whereas the reverse is true after reading a word associated with down (such as "basement" or "bleak"). This occurs for both concrete and abstract words. This ability of the words to orient attention in space is known as conceptual cueing. Conceptual cueing not only affects target response efficiency, it can also affect 
saccade trajectories. That is, when participants' task is to move their eyes from the center of the screen to a horizontally displaced target, the path of their eye movement will veer upwards after exposure to a word associated with up, and downwards after reading a word associated with down (Dudschig, et al., 2013; Dunn, Kamide, \& Scheepers, 2014; Gozli, Chow, et al., 2013). In short, the meaning of the words has a potent impact on visual-attentional processes.

Despite the striking patterns of results produced in this line of research, it has been plagued by a significant methodological limitation: the classification of words as associated with either up or down has relied almost exclusively on researchers' intuitions. While there appears to be agreement on some items, others have been differently classified by different researchers (e.g., "mosquito" has been classed as both $u p$ and down by different researchers). With no recourse to an objective measure, there is no way of resolving such discrepancies. Moreover, it is scientifically unsatisfying to lack an objective criterion for classification for experimental stimuli. Where researchers have reported having items rated, these are typically done with a small number of people on a small number of items, and merely to support the categorical classifications of the researchers. The purpose of the present study, therefore, was to provide researchers with an extensive of database of items rated by a large number of participants to use in this line of research. This approach allowed us to provide a continuous measure of the extent to which each of the 498 items people associated with "up" versus "down," such that in future researchers can select items with particular magnitudes of associations. It is anticipated this database will provide a more precise tool for further studies to disentangle the competing theoretical explanations for conceptual cueing (e.g., perceptual simulation, conceptual metaphor theory, language-based explanations). This is important because resolving such theoretical debates will provide important insight into the nature of the interaction between language and attention that underlies conceptual cueing effects.

\section{Method}

\section{Participants}

Participants were 180 undergraduate students from the Australian National University (average age $=21.4$ years).

\section{Item selection}

A total of 498 items were compiled. Of these, 364 were collated from items used in previous studies (Ansorge, et al., 2013; Chasteen, et al., 2010; Dudschig, et al., 2013; Estes, et al., 2008; Goodhew, McGaw, \& Kidd, 2014; Gozli, Chasteen, \& Pratt, 2013; Gozli, Chow, et al., 2013; Meier \&
Robinson, 2004; Setic \& Domijan, 2007), and were items classified as having associations with up, down, or no clear vertical spatial association (neutral). A further 134 items were included that we developed and selected as likely to have associations with up, down, or no clear vertical spatial associations.

\section{Procedure}

The 498 items were split into two 250-item sets (note that items 'car' and 'light' appeared in both sets) and participants were given one of these sets to rate. Ratings were completed with pen and paper. For each item, participants were asked: "For each word, please circle whether you associate it more with the spatial dimension of 'up' or 'down.' There are no correct or incorrect answers; we are interested in your responses. Please take care to complete the task meaningfully." Each item was placed on a separate row. For example:

\begin{tabular}{lll}
\hline Lucky & Upwn
\end{tabular}

In order to avoid issues of response bias, the ratings were forced choice. That is, there was no "neutral" or "don't know" option available. We reasoned that for items that are not systematically associated with either up or down, responses should average out at $50 \%$ of ratings with up and $50 \%$ with down. In contrast, words that do have systematic associations with vertical space should show much higher proportions with either up or down.

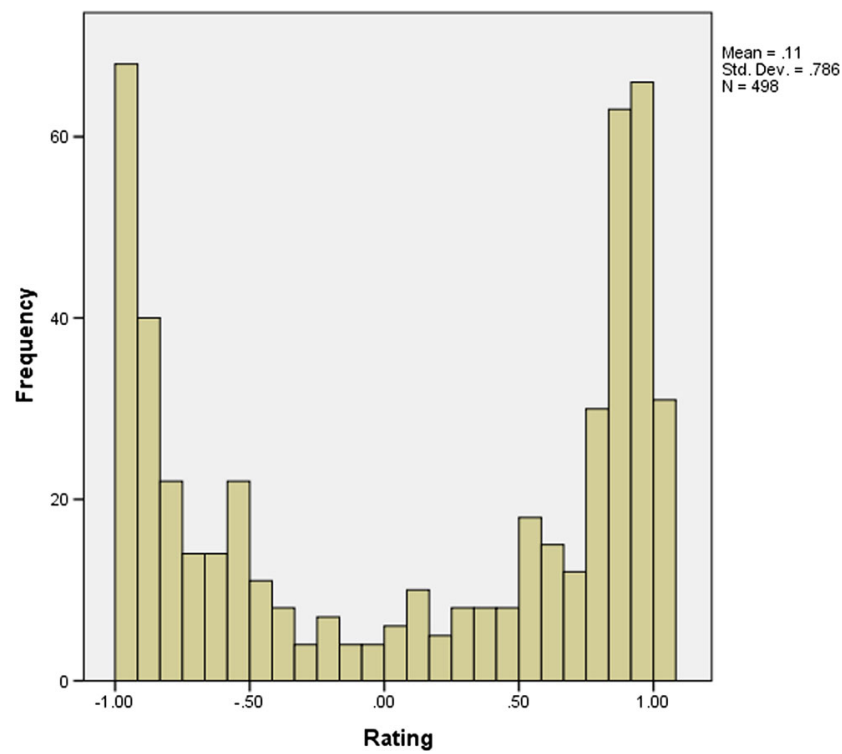

Fig. 1 Illustration of the frequency (number of words) associated with each average rating value. $-1=$ invariably associated with down by all participants, $+1=$ invariably associated with up by all participants, $0=$ no more likely to be associated with up or down, that is, $50 \%$ of responses associated with each 


\section{Results and discussion}

Scoring was done by assigning a value of -1 to responses where down was circled, and +1 to where $u p$ was circled. Missing data were exceedingly rare: participants completed on average $99.7 \%$ of the items they were asked to rate. Where missing data was present, it was simply omitted from the calculation of average ratings.

As a preliminary check, we needed to ensure that the ratings collated measured something systematic, rather than random variation. To gauge this, we examined the frequency of rating responses for each word. Random variation would amount to similar frequencies for all items, which should converge on approximately zero (indicative of $50 \%$ of responses with each dimension). In contrast, however, the ratings showed clear systematic tendencies, as illustrated in Fig. 1. Individual participant ratings for each item and summary averages can be found at: http://dx.doi.org/10.17605/OSF.IO/ N3THI

Given that the ratings were clearly tapping a systematic association between the meaning of the words and the spatial dimension, our next question was the extent to which our participants' ratings converged with the way in which researchers had previously classified items. If items were associated with up, they were assigned a value of +1 , if they were associated with neutral, they were assigned a value of 0 , and if they were associated with down, they were assigned a value of -1 . For items where different researchers had previously classified them differently, we went with the classification that was either most common or where there was not a consensus we made classification decisions. We then calculated the Pearson's correlation coefficient between the researcher's classifications of these 364 previously used items and participants' ratings, which revealed considerable agreement: $r=$ $.87, p<.001$. This suggests that researchers' intuitions do appear to be gauging something meaningful that corresponds with the way in participants classify items. What might this commonality be? One possibility, which has been gaining increasing traction, is that language-use patterns play a pivotal role in creating and transmitting these systematic associations (Goodhew, et al., 2014; Hutchinson \& Louwerse, 2013; Louwerse, 2008; Louwerse \& Jeuniaux, 2010). In other words, "happy" is associated with up because the words "happy" and "up" systematically co-occur together in language with greater frequency than "happy" and "down."

Previously we have shown that the magnitude of collocation (co-occurrence of a concept word with the word up and above versus down and below) could predict the extent to which 24 conceptual cues systematically shifted visual attention up or down in space (Goodhew, et al., 2014). To examine the relationship between language collocation and the present ratings, we calculated the correlation between the collocation difference scores from Goodhew et al. (2014) and the ratings of those 24 items (track, street, ground, road, drain, spider, sky, head, ceiling, lid, castle, candy, delay, Satan, sad, vain, hostile, bitter, God, dream, heaven, happy, earnest, sensible). This Pearson's correlation coefficient demonstrates a significant correlation between these two variables, $r=.47, p=.022$. This further supports the notion that systematic tendencies in language play a pivotal role in the transmission of these concept-space associations. In other words, patterns of language use are a strong predictor of the impact of concepts on our visual attention.

In conclusion, conceptual cueing sits at the exciting intersection of research on visual attention and language. A greater theoretical understanding of the mechanisms that underlie these systematic spatial associations and their effect on attention will provide significant insight. The purpose of the present study was to develop a sharper, more refined tool for this purpose. Here we provide the first systematic database of conceptual cues: 498 items ranked according to the average rating of their association with up and down spatial dimensions. These ratings are intended to be useful for disentangling competing theoretical accounts for conceptual cueing, such as perceptual simulation versus language association theory.

Acknowledgments This research was supported by an Australian Research Council (ARC) Discovery Early Career Research Award (DE140101734) awarded to S.C.G, and E.K. is supported by the Australian Research Council Centre of Excellence grant (CE140100041). The authors thank Erin Walsh and Reuben Rideaux for assistance with data entry.

\section{References}

Ansorge, U., Khalid, S., \& Konig, P. (2013). Space-valence priming with subliminal and supraliminal words. Frontiers in Psychology, 4, 81. doi:10.3389/fpsyg.2013.00081

Boroditsky, L., Fuhrman, O., \& McCormick, K. (2011). Do English and Mandarin speakers think about time differently? Cognition, 118(1), 126-132. doi:10.1016/j.cognition.2010.09.010

Chasteen, A. L., Burdzy, D. C., \& Pratt, J. (2010). Thinking of God moves attention. Neuropsychologia, 48(2), 627-630. doi:10.1016/ j.neuropsychologia.2009.09.029

Dudschig, C., De la Vega, I., \& Kaup, B. (2015). What's up? Emotionspecific activation of vertical space during language processing. Acta Psychologica, 156, 143-155. doi:10.1016/j.actpsy.2014.09. 015

Dudschig, C., Souman, J., Lachmair, M., de la Vega, I., \& Kaup, B. (2013). Reading "sun" and looking up: The influence of language on saccadic eye movements in the vertical dimension. Plos One, 8(2). doi:10.1371/journal.pone.0056872

Dunn, B. M., Kamide, Y., \& Scheepers, C. (2014). Hearing "moon" and looking up: Word-related spatial associations facilitate saccades to congruent locations. CogSci Proceedings.

Estes, Z., Verges, M., \& Barsalou, L. W. (2008). Head up, foot down: Object words orient attention to the objects' typical location. Psychological Science, 19(2), 93-97. doi:10.1111/j.1467-9280. 2008.02051.x 
Fischer, M. H., Castel, A. D., Dodd, M. D., \& Pratt, J. (2003). Perceiving numbers causes spatial shifts of attention. Nature Neuroscience, 6(6), 555-556.

Goodhew, S. C., Edwards, M., Ferber, S., \& Pratt, J. (2015). Altered visual perception near the hands: A critical review of attentional and neurophysiological models. Neuroscience \& Biobehavioral Reviews, 55, 223-233. doi:10.1016/j.neubiorev.2015.05.006

Goodhew, S. C., Gozli, D. G., Ferber, S., \& Pratt, J. (2013). Reduced temporal fusion in near-hand space. Psychological Science, 24(6), 891-900. doi:10.1177/0956797612463402

Goodhew, S. C., McGaw, B., \& Kidd, E. (2014). Why is the sunny side always up? Explaining the spatial mapping of concepts by language use. Psychonomic Bulletin \& Review, 21(5), 1287-1293. doi:10. 3758/s13423-014-0593-6

Gozli, D. G., Chasteen, A. L., \& Pratt, J. (2013a). The cost and benefit of implicit conceptual cues for visual attention. Journal of Experimental Psychology: General. doi:10.1037/a0030362

Gozli, D. G., Chow, A., Chasteen, A. L., \& Pratt, J. (2013b). Valence and vertical space: Saccade trajectory deviations reveal metaphorical spatial activation. Visual Cognition, 21(5), 628-646. doi:10.1080/ 13506285.2013.815680

Hutchinson, S., \& Louwerse, M. M. (2013). Language statistics explain the spatial-numerical association of response codes. Psychonomic Bulletin \& Review. doi:10.3758/s13423-013-0492-2

Louwerse, M. M. (2008). Embodied relations are encoded in language. Psychonomic Bulletin \& Review, 15(4), 838-844. doi:10.3758/PBR. 15.4.838
Louwerse, M. M., \& Jeuniaux, P. (2010). The linguistic and embodied nature of conceptual processing. Cognition, 114(1), 96-104. doi:10. 1016/j.cognition.2009.09.002

Maguire, E. A., Valentine, E. R., Wilding, J. M., \& Kapur, N. (2003). Routes to remembering: The brains behind superior memory. Nature Neuroscience, 6(1), 90-95. doi:10.1038/nn988

Meier, B. P., \& Robinson, M. D. (2004). Why the sunny side is up: Associations between affect and vertical position. Psychological Science, 15(4), 243-247. doi:10.1111/j.0956-7976.2004.00659.x

Santiago, J., Lupianez, J., Perez, E., \& Funes, M. J. (2007). Time (also) flies from left to right. Psychonomic Bulletin \& Review, 14(3), 512516. doi:10.3758/BF03194099

Setic, M., \& Domijan, D. (2007). The influence of vertical spatial orientation on property verification. Language and Cognitive Processes, 22(2), 297-312. doi:10.1080/01690960600732430

Thomas, L. E., \& Lleras, A. (2007). Moving eyes and moving thought: On the spatial compatibility between eye movements and cognition. Psychonomic Bulletin \& Review, 14, 663-668.

Thomas, L. E., \& Lleras, A. (2009). Swinging into thought: Directed movement guides insight into problem solving. Psychonomic Bulletin \& Review, 16, 719-723.

Weger, U. W., \& Pratt, J. (2008). Time flies like an arrow: Space-time compatibility effects suggest the use of a mental timeline. Psychonomic Bulletin \& Review, 15(2), 426-430. doi:10.3758/ PBR.15.2.426

Zwaan, R. A., \& Yaxley, R. H. (2003). Spatial iconicity affects semantic relatedness judgements. Psychonomic Bulletin \& Review, 10(4), 954-958. doi:10.3758/BF03196557 